\title{
R Research Sourare \\ Vulnerability and Adaption of Power Grids for Global Warming Induced Energy Demands
}

\author{
Kishan Prudhvi Guddanti \\ Arizona State University https://orcid.org/0000-0002-0041-8584 \\ Yang Weng ( $\square$ yang.weng@asu.edu ) \\ Arizona State University \\ Yang Yu \\ Tsinghua University
}

Article

Keywords: global warming, climate change, energy demand, power grid

Posted Date: October 25th, 2021

DOI: https://doi.org/10.21203/rs.3.rs-956921/v1

License: (1) This work is licensed under a Creative Commons Attribution 4.0 International License.

Read Full License 


\title{
Vulnerability and Adaption of Power Grids for Global Warming Induced Energy Demands
}

\author{
Kishan Prudhvi Guddanti ${ }^{1}$, Yang Weng ${ }^{*}, 1$, and Yang $\mathrm{Yu}^{2}$
}

\begin{abstract}
Global climate change not only causes extreme weather events but also impacts the consumer's energy demand, posing a concern for the safe and reliable operation of power grids. Furthermore, the high adaption rate of electric vehicles makes energy transactions volatile. Hence, it is crucial to evaluate the power grid's reliability using risk assessment studies. Here, we solve this open problem to predict the risk of blackout by developing a real-world data-driven probabilistic framework in conjunction with an expert-based power grid methodology. Utilizing the real-world energy demands, climate station's temperature data, and IPCC regional climate models, the risk of a city in California increases up to $8 \%$ in the summer of 2100. The winter, spring, summer, and fall seasons' risks also follow an increasing trend from 2000 to 2100 . We also show that a better localization planning of load hot spots like EV charging stations reduces the risk by $1.8 \%$.
\end{abstract}

\section{BACKGROUND}

$\mathbf{S}$ ustainability of the urban system is dependent on the ability of the distribution grid (DG) system to adapt to the global climate change (GCC). Since DGs form the core of the urban infrastructure, it is very hard to redevelop them to follow the unusual energy demand influenced by GCC. Hence, there is a need for the existing DGs to adapt to the future climate change scenarios [1]. Thus, DG's adaptability to GCC is reflected by the probability of the peak electricity demand exceeding the load hosting capacity of the DG during the progression of GCC. The GCC progression has been squeezing the DG system by two mechanisms: raising the level and duration of peak demand in the summer season while weakening the DG's physical capability of serving. The existing literature also demonstrates that the peak load of electricity consumption will significantly inflate when GCC increases the summer temperature [2]. A sequence of studies have predicted that the urban electricity consumption during summer peak-demand hours will significantly surge [2]-[5]. The expected higher summer temperature will also raise the fault probabilities of DG's grid and the associated devices. However, the statistics and literature have demonstrated that most of the major DG blackout accidents in the world are driven by the consumption peak [4], [6].

Assessing the overall risk of an entire urban DG is urgent. The progress of GCC has pushed up the average temperature every year, and 20 to $40 \%$ of the global population have experienced over $1.5 \mathrm{deg}$ warming in at least one season [7]. GCC also expanded the number of cooling-degree days from 180 days in 2000 to 210 days in 2020 . According to the prediction from [5], the number of cooling degree days will continue to grow to 570 days by 2070 if the regional climate scenario (REMO) occurs [5]. While the current DGs were mostly developed before the year 2000 when the climate was still moderate and expected to be stable, the current DGs can be highly vulnerable during the future progression of GCC. However, there lacks a method to estimate DG's vulnerability to the GCC. The key to assessing DG's adaptivity is to accurately estimate the operation boundary of a DG's capability. For example, estimating the vulnerability of an urban infrastructure system due to GCC includes predicting the temperature rising's impact on the future energy demand and the decline of grid capacity.

The effects of global climate change on power grids are poorly understood, leaving independent system operators (ISOs) with little choice but to study mere infrastructure reliability based on historical climate conditions and associated physical equipment failure [1]. However, this infrastructure reliability-based study is only one, of many, that reviews the reasons leading to a power grid blackout/failure. It cannot predict or estimate the entire grid/system-level failures due to peak-demand increase caused by other factors like climate change, population and economic growth [8]-[10]. This system/grid-level risk assessment considers the fact that every power grid irrespective of the abundant amount of generation, has a loadability boundary which is pre-determined by its graph network-like structure and admittance of the transmission line. [11], [12] showed in their climate change study that empirically, a significant increase in temperature impacts the peak demand of the power grid. Specifically, [12] showed that an $18 \%$ increase in peak demand due to various occurrences of extreme events caused an estimated loss of 180 billion\$. However, it only discussed the increase in peak demand due to climate change, but it does not provide a model to assess the risk of a power grid blackout. [13]-[17] provide risk assessment models for power grids with the help of infrastructure reliability based methodologies. These methodologies use climate change and historical data of power grid infrastructure failure rates to assess the risk. [18] presents an approach for system restoration after the occurrence of a climate change-associated outage by oversimplifying the system to a DC formulation. However, there is still a need for the power system operators to understand the operational limits of the power grid when there are no device failures but may still result in a system/grid-level blackout [19]. This is especially true when the operators have to perform a dynamic topological reconfiguration on the power grid in real-time [20]-[22]. In this article, to enable the power grid operators, for the first time, we provide a model to study the security of grid-level failure due to climate change using both domain and statistical information.

\footnotetext{
${ }^{1}$ Department of Electrical, Computer and Energy Engineering at Arizona State University, Tempe, U.S.A. ${ }^{2}$ Institute for Interdisciplinary Information Sciences, Tsinghua University, Beijing, China. Corresponding author: Yang Weng (email: yang.weng@asu.edu).
} 


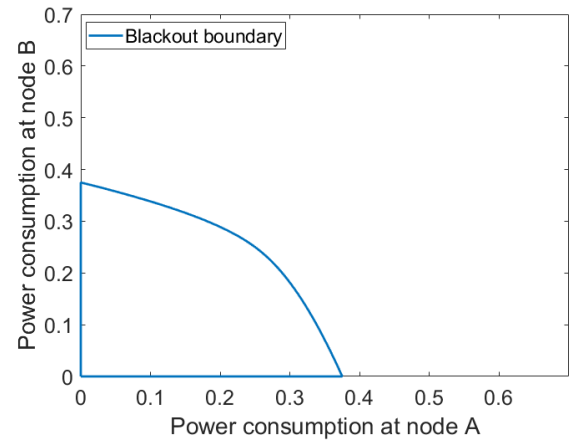

(a) Blackout boundary of the triangle shape 3 bus system in Fig. $2 \mathrm{a}$

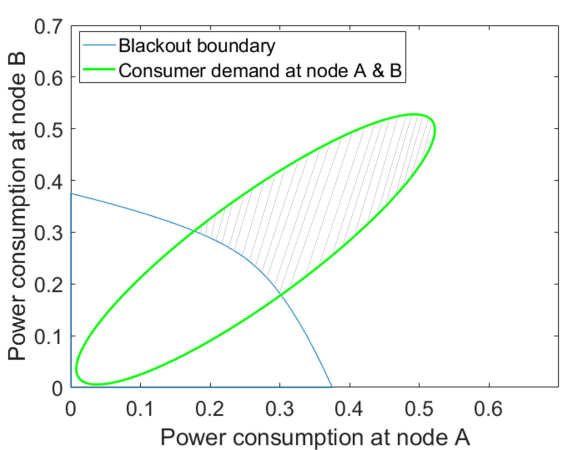

(b) Blackout boundary of the power grid and consumers' power demand.

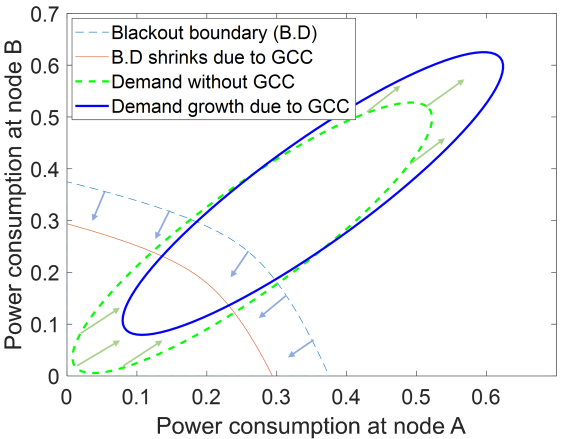

(c) Blackout boundary, consumers' power demand before and after influence of global climate change.

Fig. 1: Illustration to calculate probability of power grid blackout due to global climate change. The considered power grid is a triangle shape three bus system similar to the one in Fig. 2a except the names of nodes e.g. 1, 2, and 3 are replaced by C, A, B respectively. (a), Blackout boundary of a power grid with two consumers located at nodes A and B. If the power demand on the grid falls beyond the blue outline (blackout boundary) then the power grid collapses. (b), Blackout boundary of the power grid and consumers' power demand. The power demand of consumers is a known joint distribution indicated by the green ellipse. The ratio of the area (shaded region) of green ellipse (joint distribution) outside the blue outline (blackout boundary) to the total area of green ellipse indicates the probability of power grid collapse. (c), Impact of global climate change (GCC) on the blackout boundary of power grid and consumer's demand. Due to global climate change, the blackout boundary shrinks while the joint distribution of power demand shifts upwards (demand increases). This phenomenon of the blackout boundary and ellipse moving away from each other due to GCC causes an increase in the risk of power grid collapse.

\section{RISK OF POWER GRID COLLAPSE}

The exacerbating change in the climate causes a long-term increase in temperature. This increase in temperature results in a raise in the population's power consumption median value and burdens the power system to operate beyond its original capacity. Thus climate change deteriorates the safety and reliability of the power grid. Several risk assessment methods are proposed based on the historical failure rates of physical components (infrastructure) in the power grid. However, a well-designed power grid may have control to ensure a very low risk of infrastructure failure through redundancy, but it may still experience a system blackout due to the abnormal and less controllable nature of energy demand growth of its users. This is due to climate change or extreme events like heat waves. This abnormal energy demand growth can cause the consumers on the grid to consume more power than the maximum power transfer limits of what the power grid can withstand. Hence, it is required to first identify the "original capacity" i.e., maximum power transfer limits (watts) of the power grid which is referred to as blackout/loadability boundary and distance of the grid's operating condition to the blackout boundary as margin to collapse.

Blackout boundary: Here, we present an example to explain the risk of a power grid collapse. Let us consider a hypothetical power grid from Fig. 2a with two consumers located at nodes 2 (A) and 3 (B) respectively. The blackout boundary of this power grid is defined by its network structure and design. As shown in Fig. 1a, using the Pareto front technique ( $\Phi$ from Methods), this blackout boundary can be computed where the $\mathrm{x}$-axis and $\mathrm{y}$-axis indicate the amount of demand that the consumers at A and B can consume and any data point beyond the blackout boundary shown in Fig. 1a will result in the power grid's bankruptcy.

Risk of power grid collapse: The consumers located at nodes A and B have different amount of energy demands depending on the time of the day, season, temperature (climate change influence), and year. For example, the consumption pattern of the two consumers A and B are represented by the joint distribution typically obtained using their historical power consumption data (represented by a green ellipse in Fig. 1b. The power grid collapses when the power grid's demand due to its users on nodes $\mathrm{A}$ and $\mathrm{B}$ crosses the blackout boundary in Fig. 1a. Hence, the risk (blackout probability) is defined as the ratio of the shaded area in Fig. $1 \mathrm{~b}$ to the total area of the green ellipse. Formally, the risk or blackout probability is defined as the probability of the power grid's demand due to its users crossing the blackout boundary of the power grid. Please note that the technique presented in Methods not only calculates the blackout risk but it can also calculate the risk of power grid operating $\alpha \%$ (from Methods) closer to the power grid blackout, where $\alpha \in[0,100]$.

Impact of climate change on power grid vulnerability: The global climate change results in the increase of temperature of the earth's surface [23]. For example, the increase in temperature may cause consumers A and B to consume more power and their new increased demand consumption pattern is represented by the blue ellipse in Fig. 1c It can be observed that, when compared to the green ellipse (without climate change impact), the blue ellipse (with climate change impact) moves 
further away from the blackout boundary. Therefore, due to climate change, the area outside the blackout boundary for the blue ellipse increases more than the green ellipse which exacerbates the risk of power grid collapse. Additionally, not only the user's demand (ellipses) is impacted by the GCC but also the blackout boundary is impacted. For example, when the temperature is increased, the transmission line's conductance and susceptance change causing the power grid to weaken. As shown in Fig. 1c this results in the shrinkage of the blackout boundary. This shrinkage of the blackout boundary further moves the blackout boundary and the user's demand (ellipses) away from each other, increasing the risk of power grid vulnerability. However, in the following section we demonstrate not only how demand growth due to climate change impacts the vulnerability of the power grids but also how the localization of load centers using distributed energy resources (EV charging stations, etc.) can help the power grids to adapt to climate change-induced vulnerabilities.

III. IMPACT OF DEMAND GROWTH AND LOCATION (NODE) SENSITIVITY ON POWER GRID VULNERABILITY

Here, using mesh and tree type networks, we show how demand growth due to climate change can impact the vulnerability of power grids and how the localization of loads can help adapt the power grids to climate change by reducing the vulnerability. In later sections, we extend this demonstration to real-world cases using real-world power demand, climate change data, spatiotemporal temperature data, EV data, and larger power grids.

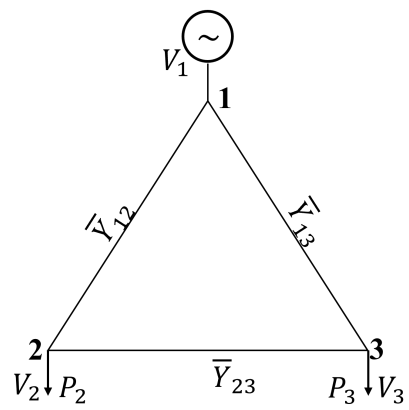

(a) 3-bus mesh network.

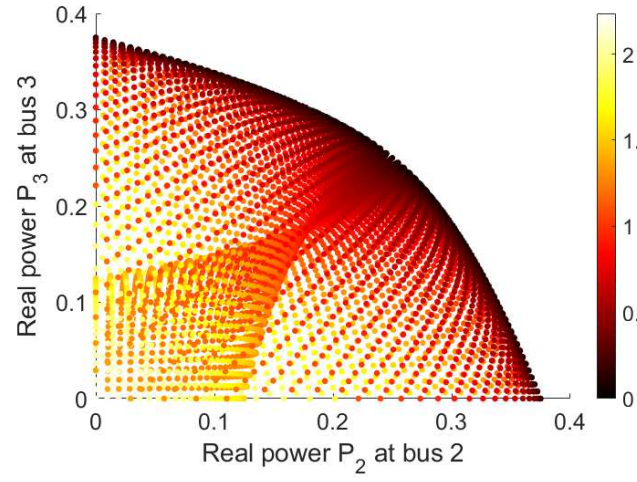

(b) Margin to collapse values for $\left(P_{2}, P_{3}\right)$ pairs.

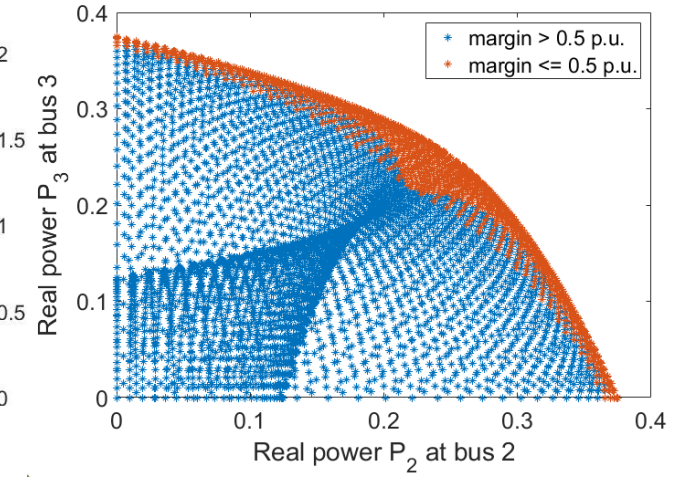

(c) Labeling of power grid operation.

Fig. 2: Mesh network example: power grid's margin values for system collapse and labeling the desired region of power grid operation. (a), A three bus mesh type network with two consumers located at nodes 2 and 3 whose demands are given by $P_{2}$ and $P_{3}$ respectively. (b), Margin to collapse value for every possible pair in $\left(P_{2}, P_{3}\right)$ plane represented using the color bar. Note that the margin to collapse values of the data points on the border are zeros. The margin to collapse values of the data points (in $\left(P_{2}, P_{3}\right)$ plane) decreases when moving towards the boundary. (c), The operation of power grid is labeled as desired and undesired operating regions by using a threshold ( $\alpha=0.5$ p.u.) on the margin to collapse values. For example, if the margin to collapse value of a operating condition (i.e., $\left(P_{2}, P_{3}\right)$ pair value) is greater than 0.5 p.u. (blue region) then it is desired and otherwise undesired (orange region). Note that when the threshold $(\alpha)$ is zero, the undesired region indicates the power grid collapse events.

System information: Specifically, the 3-bus mesh and tree networks presented in Fig. 2a and Fig. 3a are used as examples whose transmission lines are purely resistive i.e., $\bar{Y}_{12}=\bar{Y}_{23}=\bar{Y}_{13}=1 p . u$. For both mesh and tree networks, node 1 has the generator and nodes 2,3 have the users whose power demands are indicated by $P_{2}, P_{3}$ respectively.

Blackout/loadability boundaries of the mesh and tree networks: The maximum power transfer limit (blackout boundary) of a power grid is dependent on its design and network structure. Using the Pareto front technique ( $\Phi$ from Methods), the blackout/loadability boundary can be charted for mesh and tree networks as shown in Fig. 2b and Fig. $3 \mathrm{~b}$ respectively. We can observe in both Fig. $2 \mathrm{~b}$ and Fig. $3 \mathrm{~b}$, a heat map is drawn for every possible pair of $\left(P_{2}, P_{3}\right)$ and the color of each data point from color bar indicates the distance (margin) to system collapse. It can be noted that the pairs of $\left(P_{2}, P_{3}\right)$ that contribute to system collapse (located on the outer boundary of these heat maps) in Fig. $2 b$ and Fig. $3 b$ have their margin to collapse (color bar) values ( $\Phi$ values from Methods) to be zero. The operating points $\left(P_{2}, P_{3}\right)$ beyond the boundaries in Fig. $2 b$ and Fig. $3 b$ result in a blackout as the power networks cannot host any additional real power demand beyond the loadability boundary.

Safe and unsafe operating regions of power grids: In practice, power system operators are more cautious about the worstcase scenario (blackout). Hence, to be on a safer margin, the operators prefer to know the probability of power grid operating like $90 \%$ close to the blackout boundary. In this article, such an analysis is made possible for the first time, by assigning a positive threshold value (in this example 90\%) to $\alpha$ (from Methods). For example, in the case of the 3-bus mesh network in Fig. 2c the operator may want to identify the operation of the power grid as unsafe (orange region) when the consumers' demand (data points in $\left(P_{2}, P_{3}\right)$ plane) drives the margin to collapse (color bar) values to range between 0.5 p.u. to 0 p.u. (note that a 0 p.u. indicates the blackout of the power grid). In the same manner, from Fig. 2c the operation of a power grid is labeled as safe (blue region) when the consumer demand drives the margin to collapse values to be greater or equal to 0.5 


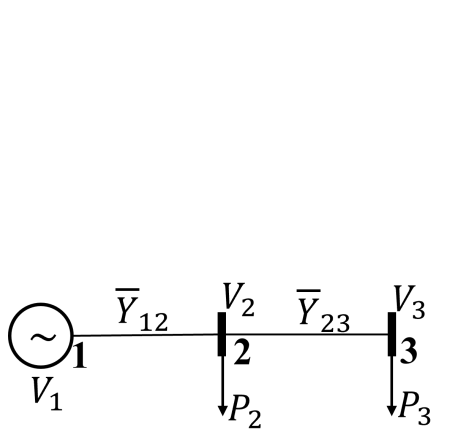

(a) 3-bus tree network

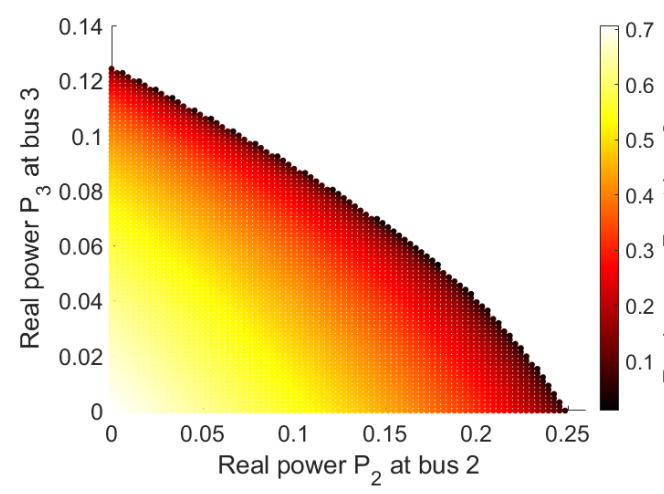

(b) Margin to collapse values for $\left(P_{2}, P_{3}\right)$ pairs.

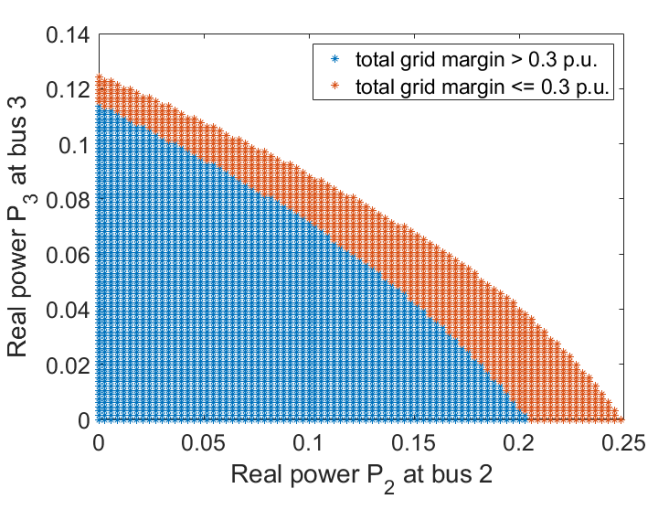

(c) Labeling of power grid operation.

Fig. 3: Tree network example: power grid's margin values for system collapse and labeling the desired region of power grid operation. (a), A three bus tree type network with two consumers located at nodes 2 and 3 whose demands are given by $P_{2}$ and $P_{3}$ respectively. (b), Margin to collapse value for every possible pair in $\left(P_{2}, P_{3}\right)$ plane represented using the color bar. Note that the margin to collapse values of the data points on the border are zeros. The margin to collapse values of the data points (in $\left(P_{2}, P_{3}\right)$ plane) decreases when moving towards the boundary. (c), The operation of power grid is labeled as desired and undesired operating regions by using a threshold $(\alpha=0.3$ p.u.) on the margin to collapse values. For example, if the margin to collapse value of a operating condition (i.e., $\left(P_{2}, P_{3}\right)$ pair value) is greater than 0.3 p.u. (blue region) then it is desired and otherwise undesired (orange region). Note that when the threshold $(\alpha)$ is zero, the undesired region indicates the power grid collapse events.

p.u. Similarly, in the case of 3-bus tree network, as shown in Fig. 3c the safety threshold value of $\alpha=0.3$ p.u. is selected. Thus, depending on the power demand (load) of the consumers, the power grid may operate in a safe or unsafe condition.

Load scenario types: For the 3-bus mesh and tree networks presented in Fig. 2a and Fig. 3a the consumers are located at nodes 2 and 3. Generally, the consumers' power demand is dependent on the temperature they are exposed to. For demonstration, in this example we directly consider three load scenario types for the 3-bus mesh and tree networks. The three load types are as follows: 1) load type 1 (from Fig. 4a, green ellipse): power consumption of two users at the temperature $T_{1}$ and among which node 3 user's power consumption is highly sensitive to temperature, while node 2 user's power consumption is less sensitive to temperature, 2) load type 2 (from Fig. 4b blue ellipse): power consumption of the same users discussed in load type 1 but at a higher temperature $T_{2}\left(T_{2}>T_{1}\right)$. We can observe from Fig. $4 \mathrm{~b}$ that as the temperature increases to $T_{2}$, the consumer located at node 3 tends to consume more power while the consumer at node 2 tends to consume less power, and 3) load type 3 (from Fig. 4c magenta ellipse): In this setup, we consider same power consumption behaviors of the two users from load type 2 (at temperature $T_{2}$ ) but the locations (nodes) of the consumers are swapped. For example, the temperature sensitive consumer is placed at node 2 while the less temperature sensitive consumer is placed at node 3 . Hence, for load type 3, we observe that the consumer at node 2 consumes more power while consumer at node 3 consumes less power. Thus, by comparing the calculated blackout risks of load types 1,2 , and 3, we can infer that 1) weather sensitivity (load type 1 versus load type 2 i.e., demand growth) and 2) the location of a consumer (load type 2 versus load type 3 i.e., node sensitivity/localization) impacts the risk of the power grid blackout.

Power grid vulnerability assessment: Here, we calculate the risk of a power grid operating in an unsafe region $(\alpha<0.5$ p.u. and $\alpha<0.3$ p.u. for mesh and tree networks respectively). For example, Fig. 4e presents the overlap of the 3-bus mesh network's loadability boundary (labelled with safe and unsafe regions) and the joint distribution of load type 1 from Fig. 4a Using the risk definition from Methods, for the 3-bus network with load type 1 consumers from Fig. 4e, the risk of the power grid operating in an unsafe region is calculated as the ratio of the number of samples from the joint distribution of load type 1 (green ellipse) that have the margin to collapse values below the unsafe margin threshold $\alpha=0.5$ p.u. (overlap between green ellipse, and orange region and beyond) to the total samples drawn from the joint distribution of load type 1 (green ellipse). A similar approach is repeated to calculate the risks for load types 2 and 3 as shown in Fig. $4 \mathrm{f}$ and Fig. $4 \mathrm{~g}$. Finally, the risks due to load types 1, 2, and 3 are also calculated for the 3-bus tree network as shown in Fig. 4i. Fig. 4j, and Fig. 4k, respectively. The results are presented in Tab I From Tab [] it can be observed that the risks of the mesh network $(62.12 \%, 89.70 \%, 89.87 \%)$ are much smaller than the risks of the tree network $(93.63 \%, 96.39 \%, 97.98 \%)$. This shows that the mesh grids are more robust because the tree networks are long and their voltages at each node drops when moving away from the generation. Thus, the tree networks are more susceptible to voltage issues when compared to that of the mesh networks, and hence mesh networks are more robust. 


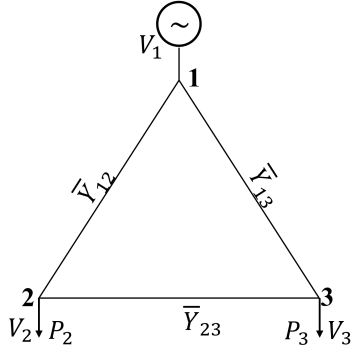

(d) 3-bus mesh network

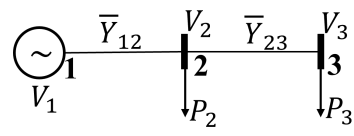

(h) 3-bus tree network

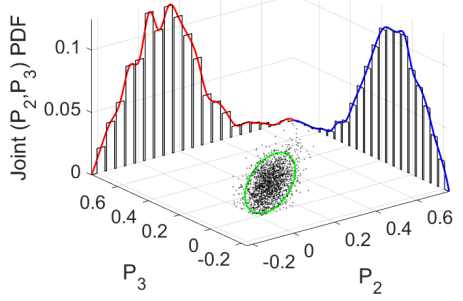

(a) Load type 1 .

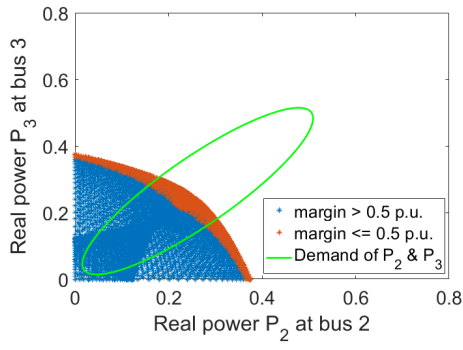

(e) Impact of load type 1 on mesh network vulnerability.

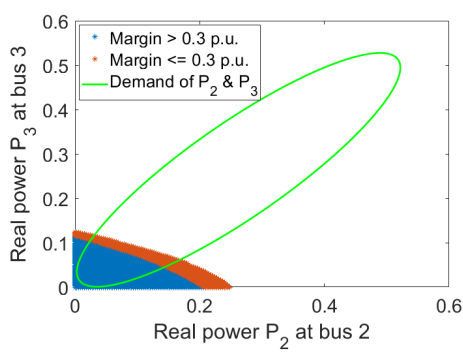

(i) Impact of load type 1 on tree network vulnerability.

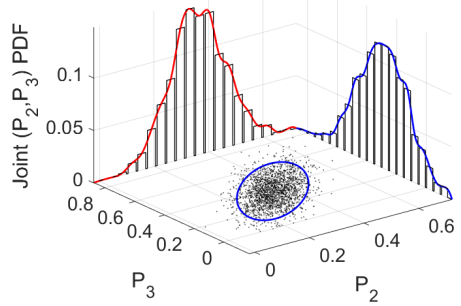

(b) Load type 2 .

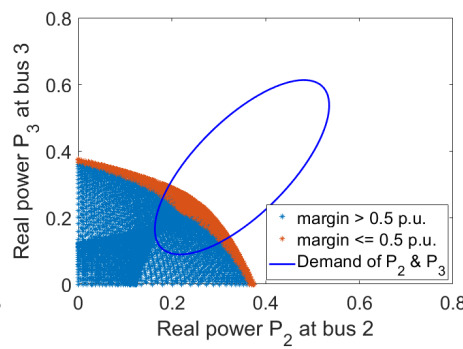

(f) Impact of load type 2 on mesh network vulnerability.

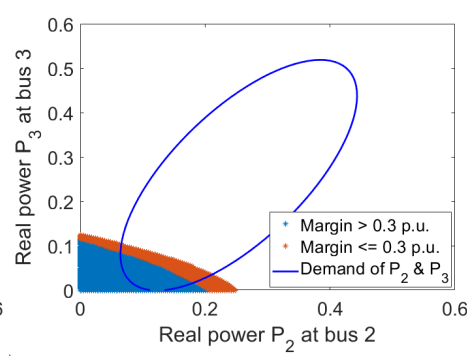

(j) Impact of load type 2 on tree network vulnerability.

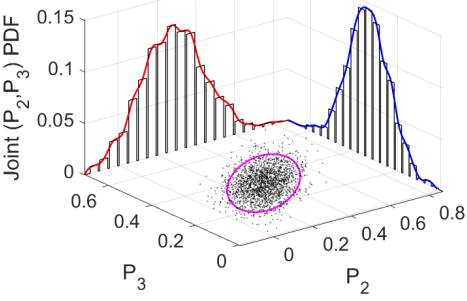

(c) Load type 3 .

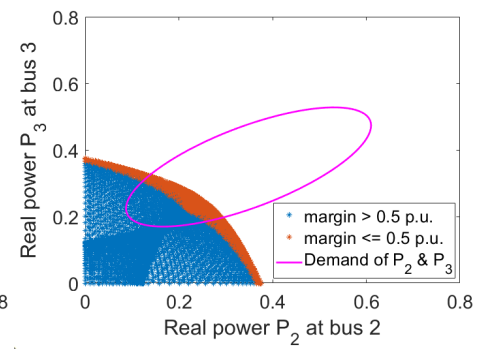

(g) Impact of load type 3 on mesh network vulnerability.

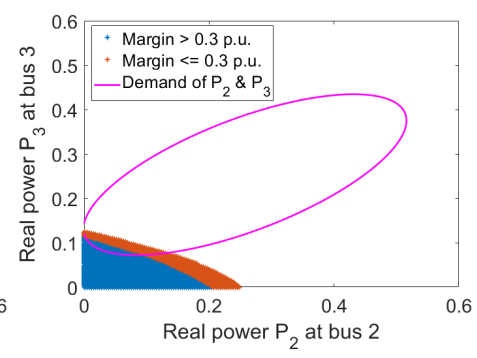

(k) Impact of load type 3 on tree network vulnerability.

Fig. 4: Dependence of power grid vulnerability on the type of the consumers' energy demand and the location of the consumers in the power grid. $P_{2}$ and $P_{3}$ are real power demands of the consumers located at nodes 2 and 3 respectively. (a), Load type 1: power demand of the consumers when temperature is $T_{1}$. Joint distribution of $\left(P_{2}, P_{3}\right) \approx \mathcal{N}(\mu, \Sigma), \mu=[0.25,0.25]$, $\Sigma=[0.0180 .016 ; 0.0160 .018]$. (b), Load type 2: power demand of the consumers when temperature is $T_{2}\left(T_{2}>T_{1}\right)$. The consumer at node 3 is more temperature sensitive than the consumer at node 2 and hence demands more power relatively. Joint distribution of $\left(P_{2}, P_{3}\right) \approx \mathcal{N}(\mu, \Sigma), \mu=[0.25,0.25], \Sigma=[0.0090 .009 ; 0.0090 .018]$. (c), Load type 3: $P_{2}$ and $P_{3}$ are real power demand at nodes 2 and 3 respectively when temperature is $T_{2}\left(T_{2}>T_{1}\right)$. Unlike load type 2 , the consumer at node 2 is more temperature sensitive than the consumer at node 3 and hence demands more power relatively. Joint distribution of $\left(P_{2}, P_{3}\right) \approx \mathcal{N}(\mu, \Sigma), \mu=[0.25,0.25], \Sigma=[0.0180 .009 ; 0.0090 .009]$. (d), A three bus mesh network with a generator at node 1, consumers with demands at nodes 2 and 3. (e), Overlap of the mesh network's margin boundary and joint distribution of load type 1 from Fig. 4a (f), Overlap of the mesh network's margin boundary and joint distribution of load type 2 from Fig. 4b (g), Overlap of the mesh network's margin boundary and joint distribution of load type 3 from Fig. 4c. (h), A three bus tree network with a generator at node 1, consumers with demands at nodes 2 and 3. (i), Overlap of the tree network's margin boundary and joint distribution of load type 1 from Fig. 4a (j), Overlap of the tree network's margin boundary and joint distribution of load type 2 from Fig. $4 \mathrm{~b}$, (k), Overlap of the tree network's margin boundary and joint distribution of load type 3 from Fig. $4 \mathrm{c}$

Climate change-induced demand growth impact on power grid vulnerability: Climate change increases the temperature of the earth which directly impacts the power demand patterns of the consumers on the power grid. This increase in temperature and therefore, an increase in power demand can be understood by comparing the load type 1 and 2 scenarios. From Tab I, it can be observed that the probability to enter an unsafe operating region increases for load type 2 (at temperature $T_{2}$ ) when compared to that of the load type 1 (at temperature $T_{1}, T_{2}>T_{1}$ ) from $62 \%$ to $89 \%$ (for mesh network) and from $93 \%$ to $96 \%$ (for tree network). This shows that the risk of the power grid is directly proportional to the power consumption sensitivity of the consumers on the grid and climate change-induced demand growth.

Adaption of power grids to climate change using localization of load centers: Similarly, from Tab I, we can also ob- 


\begin{tabular}{|l|c|c|c|c|c|l|}
\hline \hline & \multicolumn{2}{|c|}{ 3-bus mesh network } & \multicolumn{3}{c|}{ 3-bus tree network } \\
\cline { 2 - 7 } & $\begin{array}{c}\text { load } \\
\text { type 1 }\end{array}$ & $\begin{array}{c}\text { load } \\
\text { type 2 }\end{array}$ & $\begin{array}{c}\text { load } \\
\text { type 3 }\end{array}$ & $\begin{array}{c}\text { load } \\
\text { type 1 }\end{array}$ & $\begin{array}{c}\text { load } \\
\text { type 2 }\end{array}$ & $\begin{array}{l}\text { load } \\
\text { type 3 }\end{array}$ \\
\hline Unsafe & 1189 & 1786 & 1793 & 1792 & 1868 & 1895 \\
\hline Safe & 725 & 205 & 202 & 122 & 70 & 39 \\
\hline Risk & $62.12 \%$ & $89.70 \%$ & $89.87 \%$ & $93.63 \%$ & $96.39 \%$ & $97.98 \%$ \\
\hline \hline
\end{tabular}

TABLE I: Given a specific hour of the day, the probability of power grid failure due to three different load types for the 3-bus mesh and tree networks in Fig. $4 \mathrm{~d}$ and Fig. $4 \mathrm{~h}$ respectively.

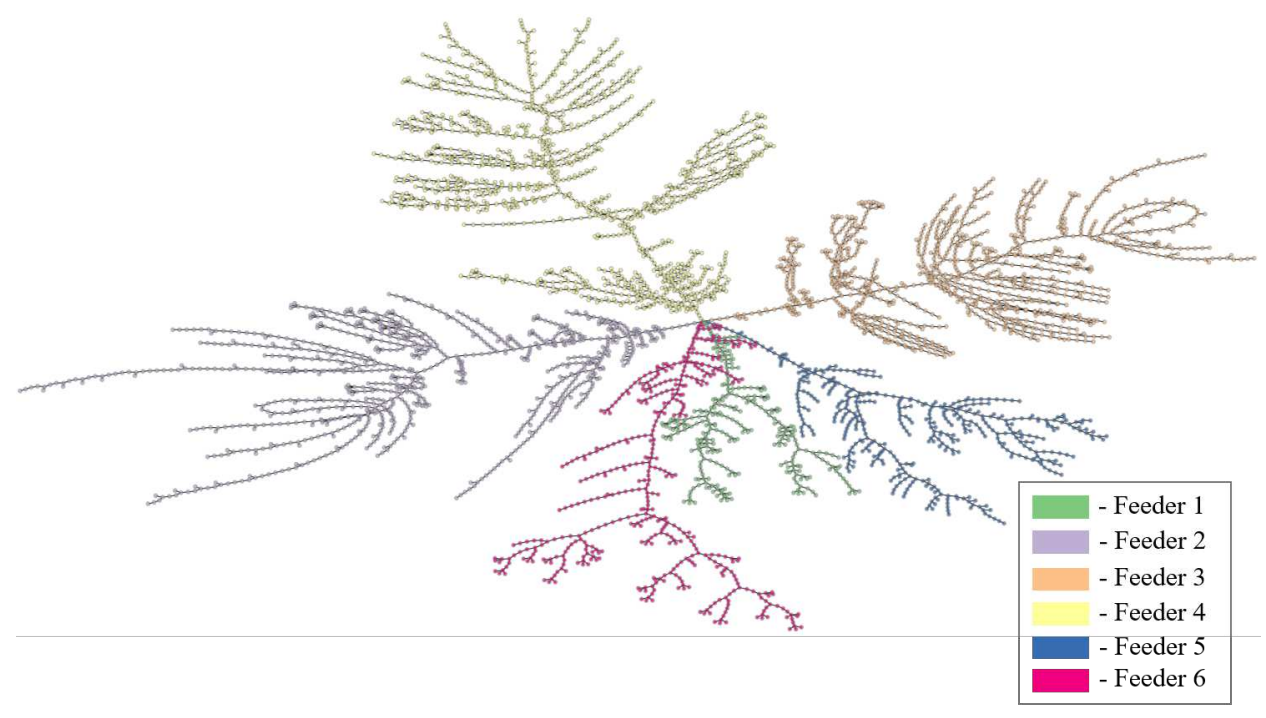

Fig. 5: The real-world distribution grid with six main feeders originating from the same substation.

serve that for load type 3 when the consumer locations are swapped (when compared to load type 2) between nodes 2 and 3 , the risk of the power grid operating in an unsafe region increased from $89.70 \%$ to $89.87 \%$ (for mesh network) and from $96.39 \%$ to $97.98 \%$ (for tree network). This signifies that different nodes have different capabilities of maintaining weathersensitive consumers and hence, it is important to assess the adaptivity planning when allocating (sanctioning) distributed energy resources on the grid.

\section{DATA}

Here, we use the approach described in the above section to analyze a modified real-world urban grid when it serves a hypothetical demand whose patterns are summarized from a real-world city. In this section, we provide the information relevant to the quality and nature of the data that is used to assess the system risk in this article.

The information required to compute the risk of the power grid are 1) the topology of the power grid along with its transmission lines' admittance values, 2) hourly power consumption data of the consumers at every node in the power grid, 3) hourly temperature data corresponding to the geo-location where the power consumption data is retrieved, and 4) the climate model which forecasts the temperature due to global climate change as a function of time.

Power grid data: The power grid information used in this article originates from a distribution system in the State of California. This information includes the topology data such as nodes and edges. It also contains the admittance values of the edges in the graph-like structure of the power grid. Fig. 5 presents the available power grid data with six different primary distribution feeders originating from a single substation. The total number of nodes on each feeder according to the lexicographic order of their feeder names are $293,953,1104,1289,440$, and 473 respectively. In this work, we present the results on analyzing the risk of Feeder 1 topology.

Load consumption data: The load consumption data corresponding to the primary distribution feeders of the grid from Fig. 5 is obtained. All the Feeders 1 through 6 originate from a single substation as shown in Fig. 5. Fig. 6a presents the aggregated sum of the real and reactive power demand of all users situated on the six feeders from 2016 to 2019 (measured from the substation side). The total real power demand split among the six feeders is presented in Fig. 6b. The consumer load profile data used in this paper belongs to the consumers in the area served by the California Independent System Operator (CAISO). A total of 83 unique load profiles at the upstream of the transformer of a real-world distribution system are used in this study. The resolution of the load consumption data is hourly, using a total of four years of data from 2015 to 2019 . All the hourly load data points for each consumer are tagged with a geolocation and a time stamp.

Feeder 1, 2, and 3 has 42, 146, 282 unique consumer databases available respectively. Since the Feeder 1 is used to analyze the proposed risk due to global warming, only the available 42 unique user database is used to assign consumers on Feeder 1. 


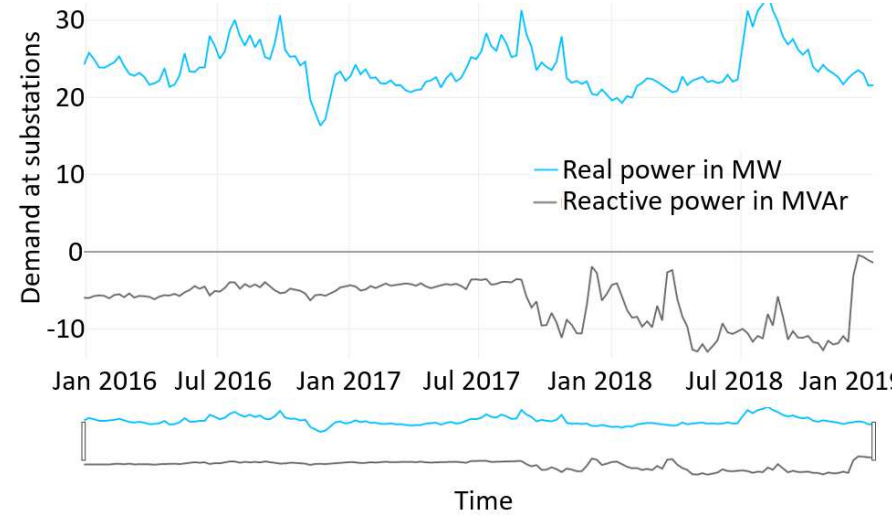

(a) The total real and reactive power demand at the origin substation of all feeders in Fig. 5 from year 2016 to 2019.

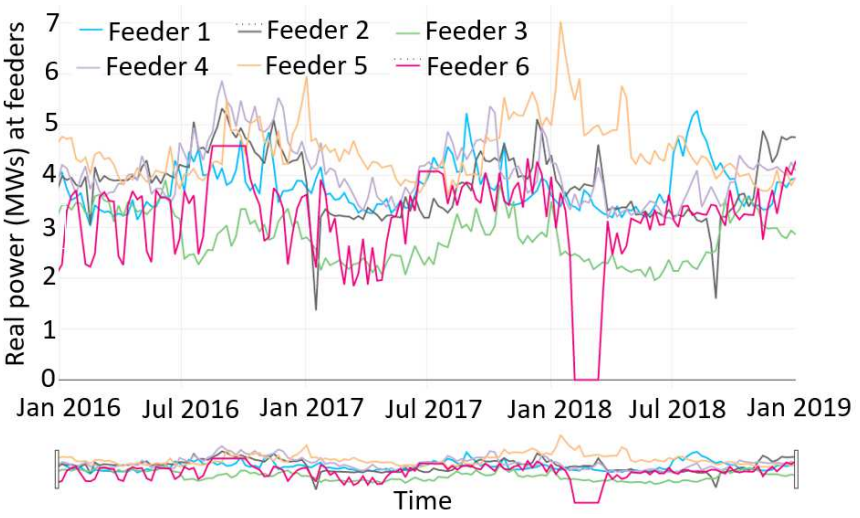

(b) The total real power demand of all the feeders from distribution grid in Fig. 5 from year 2016 to 2019.

Fig. 6: Power demand available between the years 2016 and 2019 for the distribution grid in Fig. 5
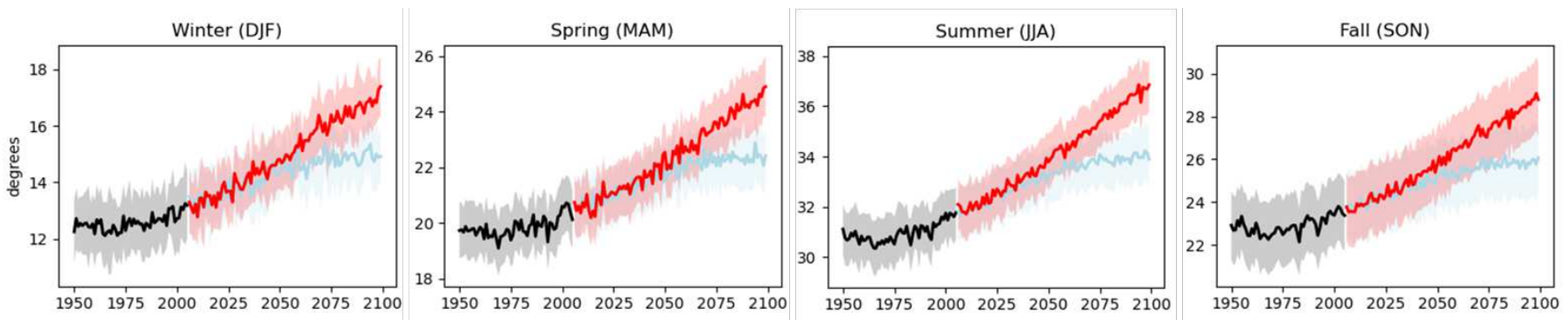

Fig. 7: Temperature forecast due to greenhouse gas emissions considering $\mathbf{R C P} 4.5$ and RCP 8.5 climate change scenarios. Seasonal average temperature forecast of California for historical (black), RCP 4.5 (blue) and RCP 8.5 (red) scenarios considering climate change [23], [25]. The historical period ends in 2005 and the forecast begins in 2006. The solid line indicates the average of 30 CMIP 5 models and their standard deviation is indicated by the corresponding shaded envelopes.

For example, Feeder 1 is a grid with 293 nodes. Each of these nodes is assigned a random user by selecting a user from the total 42 consumer database.

Historical temperature data: Using the geolocation and timestamp of the hourly load data points of each consumer from the real-world consumer database, the historical hourly temperature data experienced by each consumer at the same time from 2015 to 2019 are collected from the nearest weather stations. This is collected using a weather API developed by the National Oceanic and Atmospheric Administration (NOAA) [24].

Using the geolocation and timestamp tagged load consumption and temperature data of different consumers from real-world consumer databases and real-world weather stations of NOAA respectively, the conditional distributions of each consumer's demand given the temperature (temperature-load response curves) are learned for different consumers. The learning process of conditional distribution is further discussed in detail, later.

Climate model: Even though the historical temperature data from real-world weather stations of NOAA plays a crucial role in data-driven analysis, it is not sufficient to forecast the vulnerabilities of the power grid due to the emission of greenhouse gases i.e., global warming. To anticipate the vulnerability of the power grid accurately, a temperature forecasting model that uses the information about global warming causing factors is necessary. To address this, two forecasting models are used, one for the short-term and the other for the long-term temperature forecasting. In the short-term forecasting of temperature, it is dominantly affected by the time of the day; hence NOAA weather API is used to obtain the short-term temperature forecast data [24]. In the long-term forecasting of temperature, the temperature is significantly affected by global warming and thereby the mean of the long-term data forecast changes. We use the representative concentration pathway (RCP) scenarios that consider the impact of global warming to forecast the long-term temperature data as shown in Fig. 7] [23], [25]. Both of the models obtained correspond to the geolocation of the real-world power grid locations in California. This spatiotemporal analysis accurately models climate change both in short-term and long-term cases as a function of time and expected global warming emissions respectively.

Electric vehicle charging data: The power grid is not only sensitive to the temperature dependent energy demand response of the consumers, but it is also sensitive to the spatial effects. Here, we discuss the EV demand data that impacts the energy demand of the power grid by considering factors such as the location of prominent communities with EVs, charging stations, 


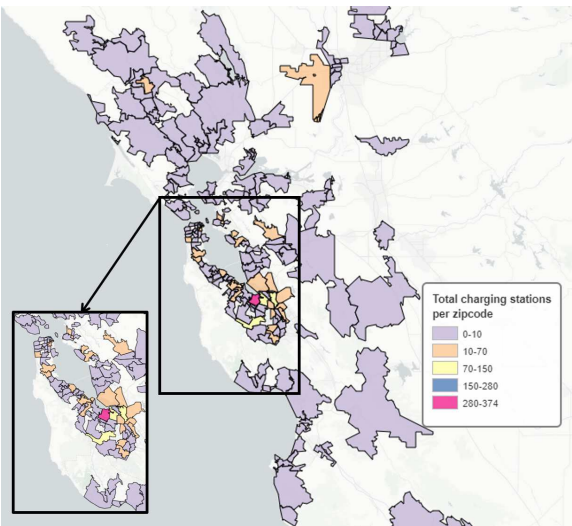

(a) The total number of charging stations in each zip code location.

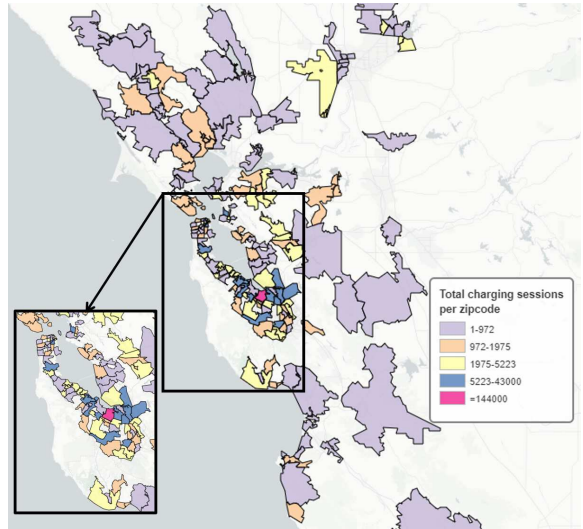

(b) The total number of charging sessions in each zip code location.

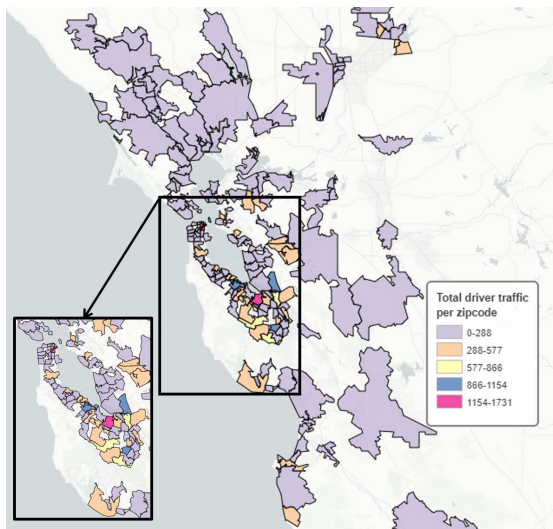

(c) Total drivers seen during an year in each zip code location.

Fig. 8: Electric vehicle data for various zip code locations. (a), the total number of charging stations in each zip code location. The inset figure shows the charging stations with a high concentration around the metropolitan area and low concentration away from the metropolitan location. (b), the total number of charging sessions (charging activity) of a year in each zip code location. The inset figure shows a high charging activity in the areas with a large number of charging stations (metropolitan area). The locations (top and bottom) with fewer charging stations have relatively high charging activity during evenings at home. This shows the unexpected growth in electric vehicle load away from metropolitan areas. (c), The total number of unique drivers that charge in each zip code location. Few locations (top and bottom) away from the metropolitan area shows the relatively high driver activity even though there are very few charging stations in those areas. This shows that the affordable prices of electric vehicles enable unexpected EV load growth.

and commute distance.

The decrease in the cost of electric vehicles along with a significant increase in the adaption rate of plug-in electric vehicles, increases the total demand on the power grid [26]. Therefore it is important to analyze the implications of the increasing number of electric vehicles on the power grid. To perform this analysis, we use the EV charging load profiles data from the year 2013. The EV charging data profiles consist of 1975 charging stations, $14289 \mathrm{EVs}$, and 589415 total charging sessions. Here, we analyze the distribution of the EV charging profiles for various locations using zip codes. We also highlight the spatial impact of EV loads.

Fig. 8 shows that the charging station infrastructure is primarily installed closer to developed metropolitan areas. However, we observe high charging activity at locations that have fewer charging stations and are far from metropolitan areas. This is supported in Fig. 8 by the observation that the total unique drivers are relatively high at the locations that are away from the metropolitan areas. This spatial nature of electric vehicles creates unexpected load hot spots on the power grid at unexpected nodes at different times of the day. This spatial factor of electric vehicles causes an irregularity and a sudden increase in demand, which will get worse as EVs are being purchased at a high rate by the consumers [26].

\section{RISK EVALUATION}

In this section, risk evaluation of the primary distribution Feeder 1 from Fig. 5 is presented. Three main inferences are made: 1) conditional distribution of power demand changes for different consumers based on the temperature and hour of the day, 2) the impact of real-world climate change-induced demand growth on power grid vulnerability, and 3) the adaption of power grids to climate change based on localization of load centers, such as distributed energy resources like electric vehicles.

Learning consumers' power consumption behavior based on temperature and hour of the day: Global warming results in an increase in the average temperature of the earth's surface [8]. This causes residential and industrial consumers to use the temperature control units for longer than usual, which ultimately stresses the power grid. So, we analyze the temperature versus load consumption response curves for all the consumers in the available data set to estimate consumer behavior. This is made possible by coalescing the power consumption data of unique users from real-world consumer databases, and the temperature data from the weather API of NOAA using their hourly resolution timestamps.

For example, Fig. 9a is an error bar plot that shows a typical daily temperature versus load consumption response of a consumer at a selected hour 2 (24-hour clock) in a year. We identify that most of the temperature response curves are parabolic in nature. These temperature response functions are highly dictated by the usage of cooling and heating units as they are major loads in households. We observe that these temperature response curves are "U" shaped and they are roughly symmetric around an equilibrium temperature value as shown in Fig. 9a. From Fig. 9a, it can be observed that as the outside temperature becomes cooler than the equilibrium temperature (minimum power demand point of the consumer), the power consumption increases indicating the usage of heating units. Similarly, when the outside temperature becomes hotter than the equilibrium temperature, then the power consumption of the user increases indicating the usage of cooling units. This suggests that the 


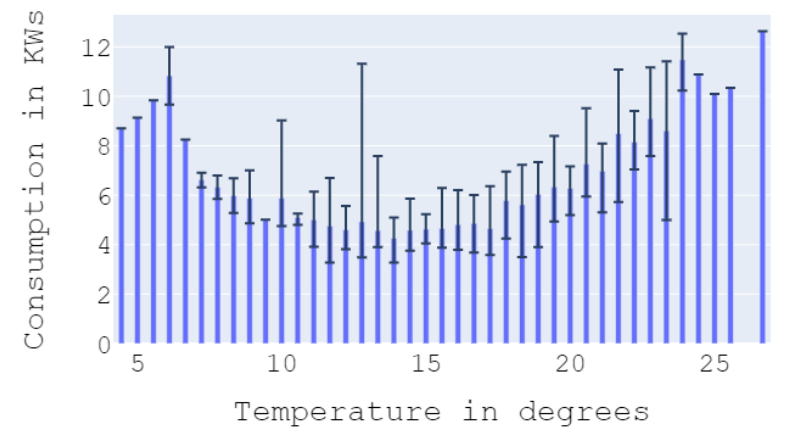

(a) Power demand versus temperature curves.

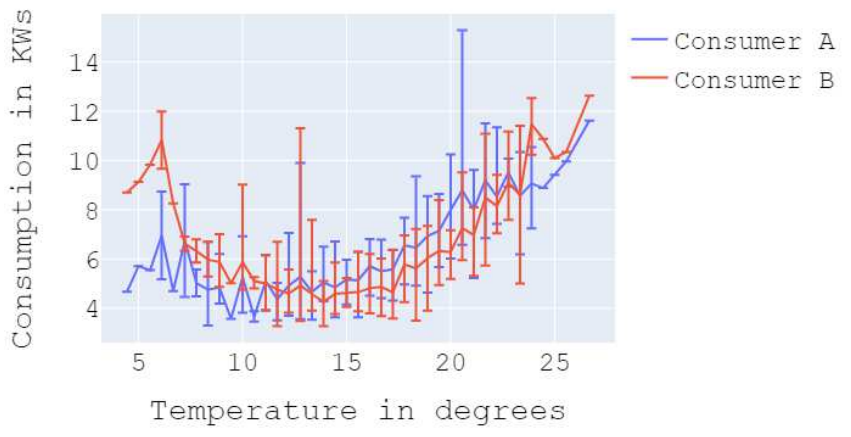

(b) Power demand versus temperature curves for two consumers.

Fig. 9: Annual average power demand of consumers versus temperature. (a), Annual average power consumption of a consumer at hour 2 of the day. These error bars represent the variability of the data where the height of the bar indicates the average power consumption of the consumer. The error line of each bar indicates the respective maximum and minimum power demand values. (b), Solid line indicates the annual average power consumption of two consumers "A" (blue) and "B" (orange) at hour 2 of the day. The error lines indicate the respective maximum and minimum power demand values. The equilibrium temperature for consumer $\mathrm{A}$ is around 10 degrees and for consumer B it is around 15 degrees. This shows that different consumers have different tolerance level to temperature.

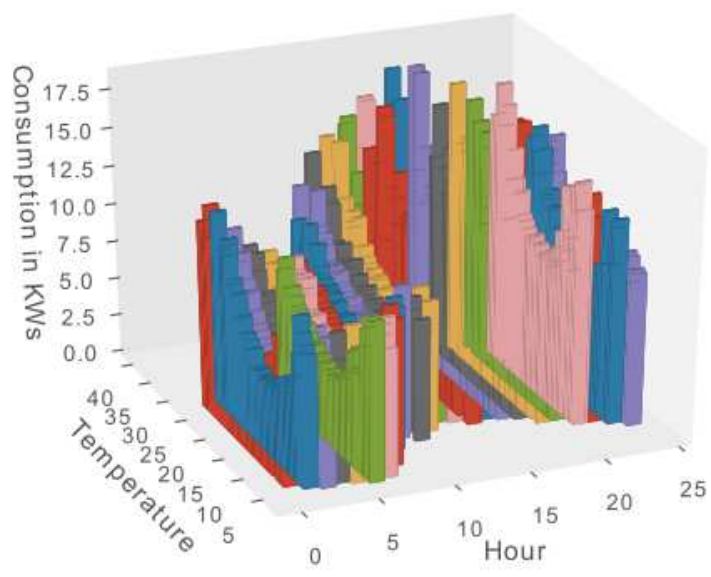

Fig. 10: Power consumption of a user with respect to the temperature and different hours of the day from an entire year.

users in the available dataset use both cooling and heating units depending on whether they feel hot or cold respectively. Later we use this knowledge of the temperature-load response function to predict the user's power consumption behavior based on temperature.

It can be inferred by looking at the equilibrium temperature value of a consumer to know when the consumer stops using the heating unit and starts using the cooling unit. For example, Fig. $9 b$ presents the temperature response curve of two different users, "A" and "B". It can be observed that the user "A" prefers to use the cooling unit than the heating unit when the temperature is greater than $10 \operatorname{deg} C$. However, the user "B" prefers to use the cooling unit rather than the heating unit when the temperature is greater than $15 \operatorname{deg} C$. Hence, we make sure to model the temperature-load response curve separately for every user depending on the user's equilibrium temperature.

However, it is not sufficient to model the power demand only conditioned on the temperature since the power demand of a consumer also depends on the time of the day. So, we identified to model the demand response functions of different consumers considering both the temperature and the time of the day. For example, Fig. 10 shows the annual average power consumption behavior of the user for the temperature and hour of the day in a year. It can be observed that at hours 12 to 15 (12pm to $3 \mathrm{pm}$ ), there are no bar plots compared to other hours of the day. This is because the noontime is hot and the bar plots at hours 12 to 15 are only half of the "U" shape indicating the usage of only cooling units. Therefore, it is important to model the demand response $\left(\boldsymbol{P}_{i}\right)$ curve for every unique user (say $\left.i\right)$ given both temperature $(t)$ and hour of the day $(h)$ i.e., $\boldsymbol{P}_{i} \mid t, h$.

The temperature values are divided into bins of width $3 \operatorname{deg} C$. The parameters of the distribution $\boldsymbol{P} \mid t, h=\left[\left(P_{1}, P_{2}, \cdots, P_{n}\right) \mid t, h\right]_{256}$ for $n$ different consumers given a selected temperature bin value $t$ and hour of the day $h$ are learned by assuming that ${ }^{257}$ 

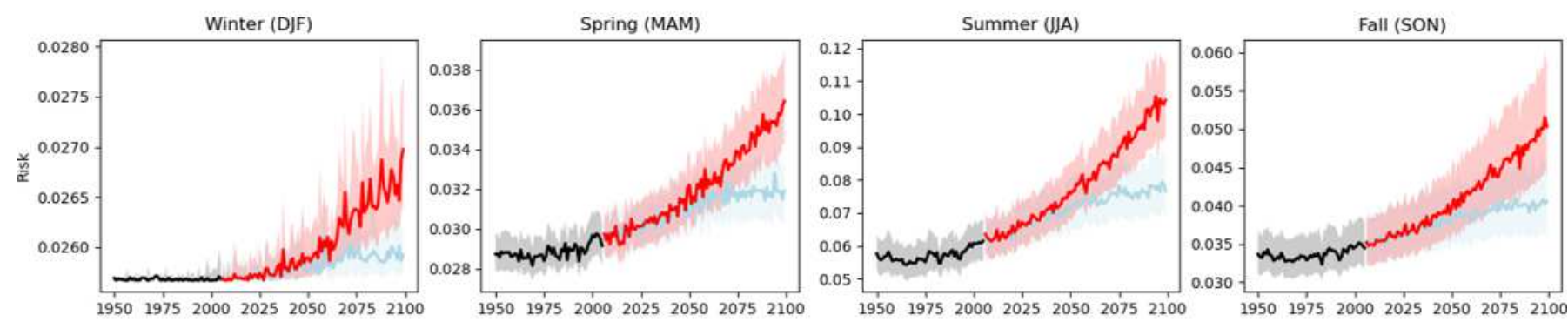

Fig. 11: Seasonal comparison of the risks for RCP 4.5, and RCP 8.5 climate change scenarios at hour 1 . The risk of power grid operation violating a threshold of $2 \mathrm{MW}$ margin to system blackout at hour 1 for Feeder 1 . The risks are calculated for the global climate change scenarios like RCP 4.5 (blue line), RCP 8.5 (red line), and historical temperature records up until 2005 (black line). The solid line indicates the average of 30 CMIP5 models and their standard deviation as indicated by the corresponding shaded envelopes.
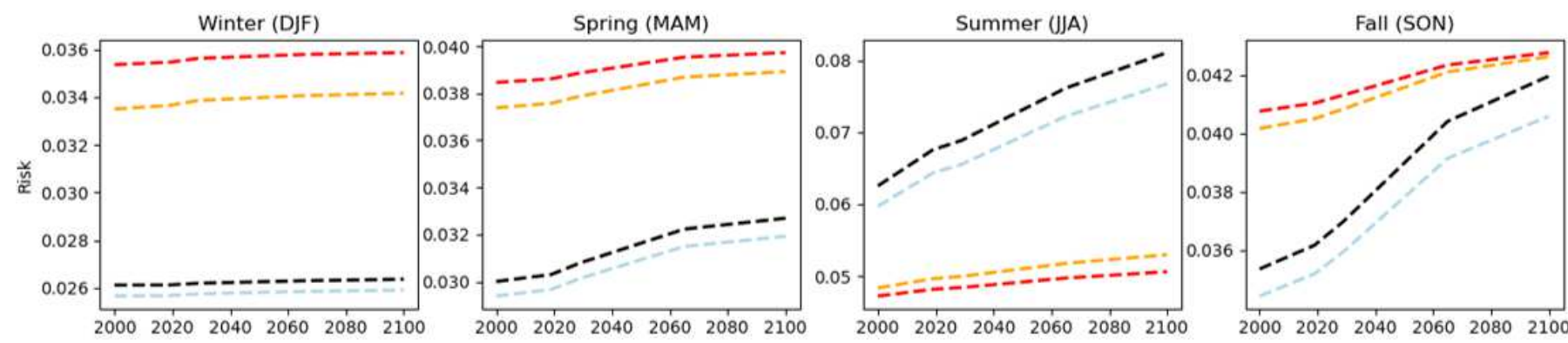

Fig. 12: Hourly comparison of the risks for RCP 4.5 climate change scenario. The risks for violating a threshold of 2 MW margin to system blackout for hours 1 (blue line), 12 (red line), 16 (orange) and 24 (black line) for Feeder 1 during the climate scenario RCP 4.5 .

$P_{1}, P_{2}, \cdots, P_{n}$ are all conditionally independent given the temperature $t$ and hour of the day $h$. Therefore, the parameters of the conditional distributions for $n$ different consumers are learned independently i.e., $\boldsymbol{P} \mid t, h=\left[\left(P_{1}, P_{2}, \cdots, P_{n}\right) \mid t, h\right]=$ $\left(P_{1} \mid t, h\right) \cdot\left(P_{2} \mid t, h\right) \cdots\left(P_{n} \mid t, h\right)$. This conditional independence is assumed to handle the incomplete datasets corresponding to the estimation of the joint distribution of total demand on power grid $(\boldsymbol{P})$ given a temperature and hour of the day. In other words, the power consumption of two households can be estimated individually based on the outside temperatures they are experiencing and the hour of the day. Finally, the parameters of each distribution of $P_{i} \mid t, h \forall i=1,2, \cdots, n$ are estimated by using a quadratic curve fitting (in a least square sense) since the shape of the temperature-load response function is " $U$ " shaped as shown in Fig. $9 \mathrm{~b}$.

Impact of real-world climate change induced demand growth on power grid vulnerability: Here, we evaluate the power grid vulnerability using the learned conditional distribution $(\boldsymbol{P} \mid T, h)$ from the real-world consumers (Fig. 6), historical spatiotemporal temperature (Fig. 10, Fig. 9), and climate model databases (Fig. 77. For example, as shown in Fig. 11, the probabilities of a power grid operating in an unsafe region (i.e., $2 \mathrm{MW}$ margin to collapse) at hour 1 of the day from the year 1950 to 2100 are calculated for the Feeder 1 . It can be observed that the risk increases by $0.4 \%$ during the period of 2000 to 2019. However, if the global warming problem is not sufficiently addressed, according to the climate model projections [23] from Fig. 7, the risk is projected to increase by $4 \%$ during the summer of year 2100 at hour 1 of the day. Furthermore, depending on the hour of the day this risk can worsen, such as noontime which is roughly the hottest time of the day. For example, Fig. 12 presents the risks for violating a $2 \mathrm{MW}$ margin to collapse at hours 1, 12, 16, and 24 of the day for the Feeder 1. It can be observed that during the winter, spring, and fall seasons, the risks of hours 12,16 are higher than that of the hours 1,24 due to the cooler temperatures. However, during the summer season, the risks of hours 12,16 are smaller than those of the hours 1,24 due to the relatively warmer temperatures in the summer season. Additionally, we can also observe the trend of increasing risk due to global warming as the years increase. Similarly, by relocating the temperature sensitive loads to less critical nodes in the power grid, we observed that the risk is reduced by $1.8 \%$ during the summer of the year 2100 . This effect is explained further in detail below.

Adaption of power grids to climate change based on localization of load centers: Here, we describe how the location of loads plays an important role on power grid security. For example, Fig. 14 presents the margin to system failure with respect to the climate change scenarios (from Fig. 7) for each node in the power grid (from Fig. 5). Using the IPCC temperature 
predictions, the mean load consumption of the consumers at all the nodes in the grid are predicted. Finally using these predicted load consumption values, the margin to collapse value is calculated for each bus using $\Phi$ from Methods. The color bar in Fig. 14 indicates the margin to system collapse at each node in the real grid from Fig. 5 . The size of each data point in the figure is downscaled as the time (years) increases. It can be observed that the power grid's margin to collapse values decrease as the years increase, due to the global warming-induced increase in energy demands. This deterioration is even worse in case of RCP 8.5 climate change scenario from Fig. 14b when compared to the RCP 4.5 climate change scenario from Fig. 14a Therefore, it is crucial to identify the critical locations of the grid that makes it vulnerable, and the proposed framework easily enables this identification.

The location of the intermittent energy resources and temperature-sensitive consumers can play an important role in the power grid security. For example, Fig. 13 shows a layered abstraction of the power grid and how localization of loads impacts the vulnerability of it. Specifically, Fig. [13a shows three layers 1) the satellite view (layer) of the distribution grid from Fig. 5 on the google map, 2) the schematic layer shows the one-line diagram of the actual topology of a selected Feeder 1, and 3) the graph layer shows the detailed nodes and edges of the selected Feeder 1. Each node in the graph layer is assigned a consumer. Using each consumer's load and temperature profile data, the specific heat loss in KW/K (Kilo Watt per Kelvin) is calculated. This $\mathrm{KW} / \mathrm{K}$ metric helps to identify the most temperature-sensitive consumers using the historical temperature and power consumption data. The specific heat loss corresponding to each consumer in the graph layers of Fig. 13a and Fig. 13b are represented using a heat map. The difference between Fig. 13a and Fig. 13b is that the consumers are relocated at different nodes (locations) in the grid. The margin to collapse values are presented for the nodes (nodes 55, 162, and 235) in Fig. 13a and Fig. 13b using both climate change RCP 4.5 and RCP 8.5 scenarios (from Fig. 77). From Fig. 13a, we can observe that the margin to collapse values for the nodes (node 162) that are closer to the substation have larger values when compared to the nodes (nodes 55 and 235) that are farther from the substation. This shows that the vulnerability of power grids is not only dependent on the amount of power consumption, but they are also dependent on the location of the loads/consumers. The nodes 55, 162, 235 in Fig. 13b have lower heat-sensitive consumers when compared to that of the consumers in Fig. 13a. We can also observe that the nodes with lower heat-sensitive consumers from Fig. $13 \mathrm{~b}$ have higher margin to collapse values when compared to that of the values in Fig. 13a. This indicates that the amount of power consumption also impacts the risk along with the location of the loads/consumers. The risks being relatively high for the nodes that are farther from the substation can also be observed here.

\section{METHODS}

Here, a Monte Carlo simulation is proposed to assess the probability of a power grid's operating condition to violate either the blackout boundary or a user-defined margin threshold to collapse due to global climate change. The global climate change is modeled using the RCP scenarios [23], [25] which provide an estimate of change in temperature due to greenhouse gas emissions.

The risk of the power grid operating in an unsafe/undesired region ( $\alpha$ watts distance close to the system blackout event) at a given hour $(h)$ of the day is given by

$$
\begin{aligned}
\text { Risk }_{h} & =\sum_{i=1}^{t} p\left(T_{i} \mid h\right) \cdot p\left(\Phi\left[\left(P_{1}, P_{2}, \cdots, P_{n}\right) \mid T_{i}, h\right] \leq \alpha\right), \\
& =\sum_{i=1}^{t} p\left(T_{i} \mid h\right) \cdot \frac{\mathcal{N}\left(\Phi\left[\boldsymbol{P} \mid T_{i}, h\right] \leq \alpha\right)}{\mathcal{N}\left(\boldsymbol{P} \mid T_{i}, h\right)} .
\end{aligned}
$$

When $\alpha=0$, equation (1) represents the probability of power grid blackout.

The overall step-by-step procedure is as follows, first given that the temperature model $(T)$ due to climate change [23], hour of the day $(h)$ are known, the conditional distribution of the total demand on power grid $(\boldsymbol{P} \mid T, h)$ is learned. Where $\boldsymbol{P}=\left[P_{1}, P_{2}, \cdots, P_{n}\right], P_{i}$ represents the power consumption of a consumer located at node $i$, and $n$ represents the total number of nodes on the power grid.

Once the distribution of $(\boldsymbol{P} \mid T, h)$ is learned, we can draw samples $\left(\mathcal{N}\left(\boldsymbol{P} \mid T_{i}, h\right)\right)$ of user's conditional power demand distribution $\left(\boldsymbol{P} \mid T_{i}, h\right)$ given a specific temperature $\left(T_{i}\right)$ from a climate scenario of $T=\left[T_{1}, T_{2}, \cdots, T_{t}\right]$, and the hour of the day $(h)$ at which the risk has to be computed.

For every sample drawn from $\mathcal{N}\left(\boldsymbol{P} \mid T_{i}, h\right)$, Pareto front technique $(\Phi)$ from [19], [27] is used to estimate the distance (margin to collapse) between the sample and the blackout boundary (for system-level margin to collapse values [27] is used and for each bus margin to collapse values [19] is used). For example, when this margin to collapse $\Phi\left[\boldsymbol{P} \mid T_{i}, h\right]$ of a sample is equal to zero then the power grid collapses i.e., $\Phi\left[\boldsymbol{P} \mid T_{i}, h\right]=0$.

The proposed framework enables the assessment of power grid security by giving the choice to the power system operators to define a safe and unsafe operating region. For example, the power system operator may define the operating region of a power grid to be safe when $\Phi[\boldsymbol{P} \mid T, h]=m>\alpha$ watts and unsafe when $\Phi[\boldsymbol{P} \mid T, h]=m \leq \alpha$ watts where $\alpha$ is a threshold in available watts that the grid can host without an outage event. Therefore, using Monte Carlo simulations, we can count the number of samples drawn from the conditional demand distribution $\left(\boldsymbol{P} \mid T_{i}, h\right)$ that drive the power grid to operate in an unsafe region $\left(\mathcal{N}\left(\Phi\left[\boldsymbol{P} \mid T_{i}, h\right] \leq \alpha\right)\right)$ and finally compute the risk as described in equation (1). 


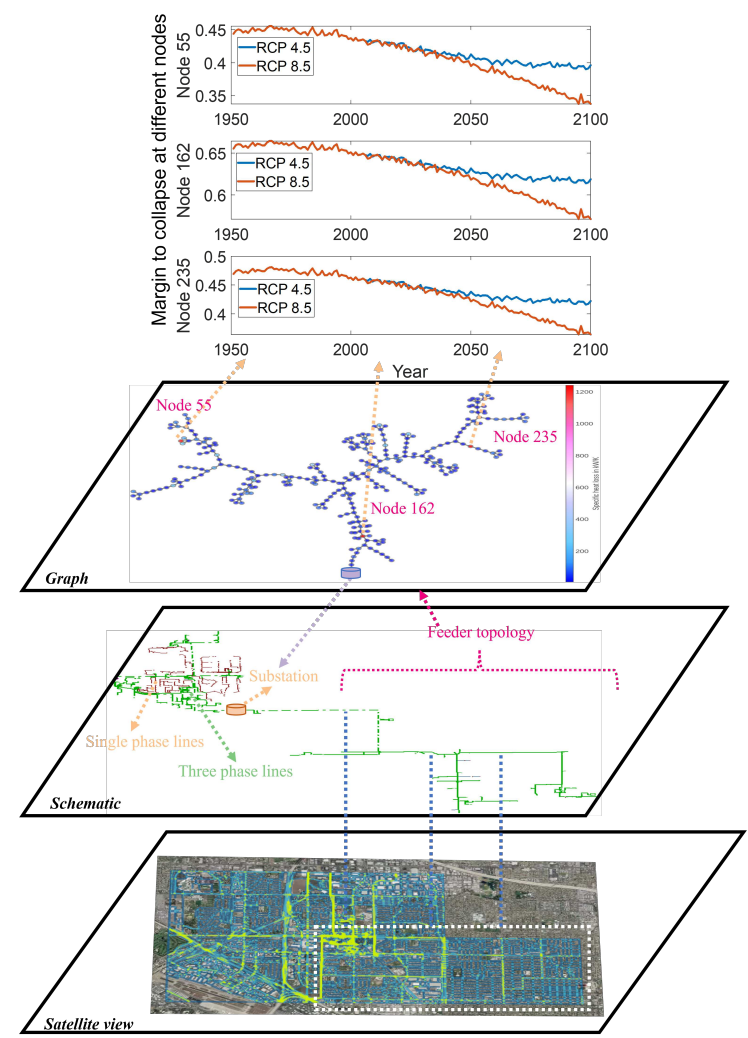

(a) Scenario when heat sensitive consumers are located at critical locations in the grid.

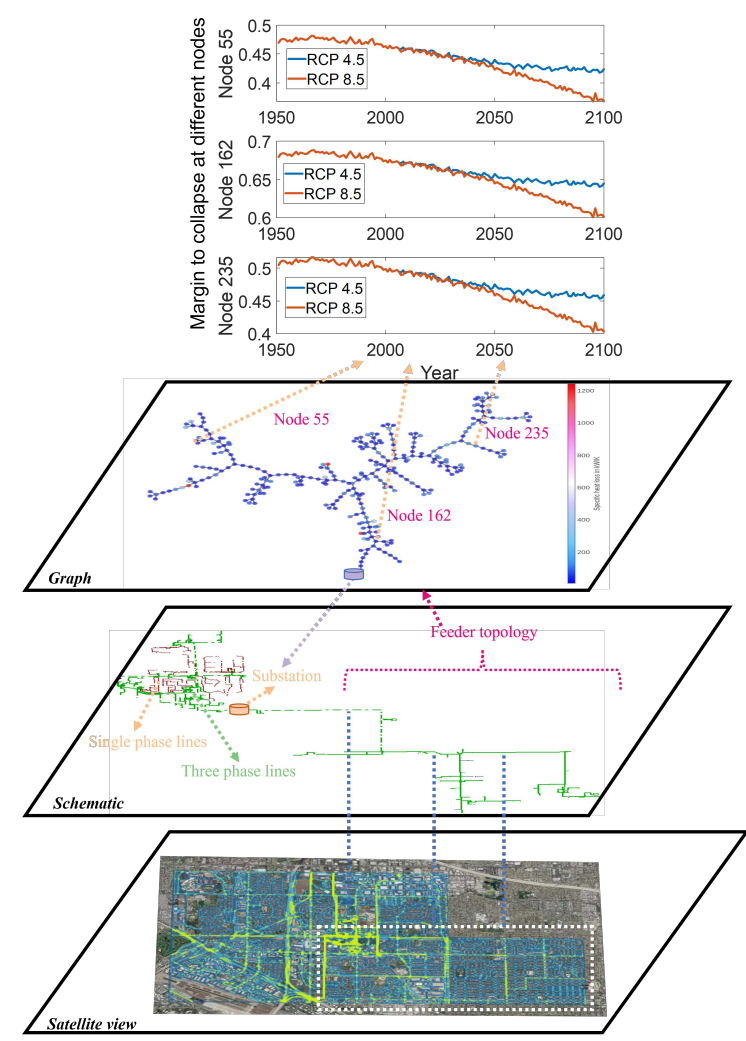

(b) Scenario when less heat sensitive consumers are located at critical locations in the grid.

Fig. 13: Impact of localization of load centers on the margin to collapse of power grids. (a), Margin to collapse when heat sensitive consumers are located at critical nodes in the grid. (b), Margin to collapse when less heat sensitive consumers are located at critical nodes in the grid.

\section{Author Contributions Statements}

All the authors contributed extensively to the work presented in this paper, including conception of the study, data identification and analysis, and drafting of the manuscript.

\section{REFERENCES}

[1] Bartos, M. D. \& Chester, M. V. Impacts of climate change on electric power supply in the western united states. Nature Climate Change 5, 748 (2015).

[2] Van Ruijven, B. J., De Cian, E. \& Wing, I. S. Amplification of future energy demand growth due to climate change. Nature communications (2019).

[3] Feron, S., Cordero, R. R., Damiani, A. \& Jackson, R. B. Climate change extremes and photovoltaic power output. Nature Sustainability (2021).

[4] Atputharajah, A. \& Saha, T. K. Power system blackouts-literature review. In International Conference on Industrial and Information Systems (2009).

[5] Matzarakis, A. \& Thomsen, F. Heating and cooling degree days as an indicator of climate change in freiburg. In Fourth Symposium on Policy and Socio-Economic Research (2009).

[6] Canizares, C. A. On bifurcations, voltage collapse and load modeling. IEEE Transactions on Power Systems 10, 512-522 (1995).

[7] IPCC. Summary for Policymakers, book section SPM, 1-30 (Cambridge University Press, Cambridge, United Kingdom and New York, NY, USA, 2013). URL www.climatechange2013.org

[8] Muir, A. \& Lopatto, J. Final report on the august 14, 2003 blackout in the united states and canada: Causes and recommendations (2004).

[9] Liu, C. Q. A discussion of the wscc 2 july 1996 outages. IEEE Power Engineering Review 18, 60-61 (1998).

[10] The New York Times. Amid heat wave in new york, 50000 lose electricity (2019). URL https://www.nytimes.com/2019/07/21/nyregion/nyc-heat-wave. html

[11] Rose, S. et al. Understanding the social cost of carbon: A technical assessment. EPRI Report 3002004657 (2014).

[12] Auffhammer, M., Baylis, P. \& Hausman, C. H. Climate change is projected to have severe impacts on the frequency and intensity of peak electricity demand across the united states. Proceedings of the National Academy of Sciences 114, 1886-1891 (2017). URL https://www.pnas.org/content/114/8/1886 https://www.pnas.org/content/114/8/1886.full.pdf

[13] Rios, M. A., Kirschen, D. S., Jayaweera, D., Nedic, D. P. \& Allan, R. N. Value of security: modeling time-dependent phenomena and weather conditions IEEE Transactions on Power Systems 17, 543-548 (2002).

[14] Cardell, J. et al. The electric power industry and climate change: issues and research possibilities. TPWRS-00710 (2007).

[15] Onen, A. Model-based grid modernization economic evaluation framework. Ph.D. thesis, Virginia Tech (2014).

[16] Vose, R. S. et al. Monitoring and understanding changes in extremes: Extratropical storms, winds, and waves. Bulletin of the American Meteorological Society 95, 377-386 (2014).

[17] Yao, R. \& Sun, K. Towards simulation and risk assessment of weather-related cascading outages. arXiv preprint arXiv:1705.01671 (2017).

[18] Cadini, F., Agliardi, G. L. \& Zio, E. A modeling and simulation framework for the reliability/availability assessment of a power transmission grid subject to cascading failures under extreme weather conditions. Applied energy 185, 267-279 (2017).

[19] Guddanti, K. P., Matavalam, A. R. R. \& Weng, Y. PMU-based distributed non-iterative algorithm for real-time voltage stability monitoring. IEEE Trans. on Smart Grid (2020). 


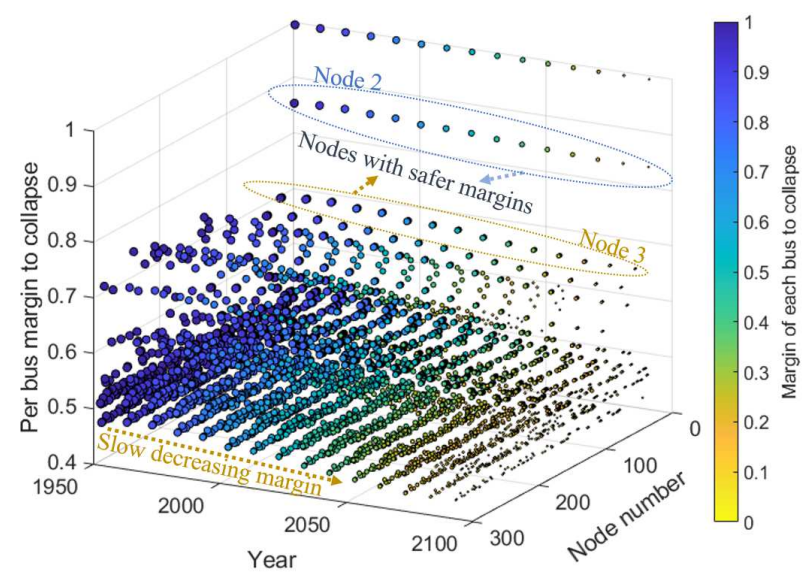

(a) Margin of each bus to system collapse corresponding to temperature projection (RCP 4.5 - blue line) from Fig. 7

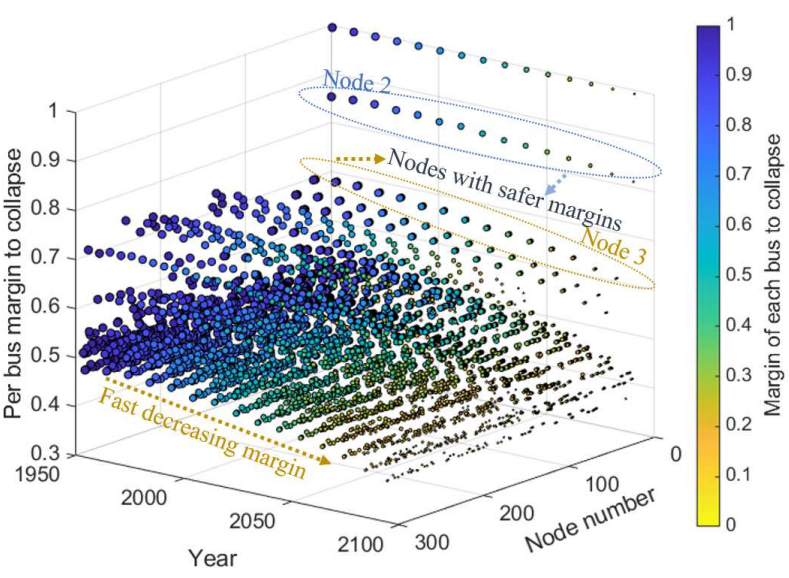

(b) Margin of each bus to system collapse corresponding to temperature projection (RCP 8.5 - red line) from Fig. 7

Fig. 14: Margin to system failure for each node in the real distribution grid with respect to time. The climate change scenarios shown in Fig. 7 are used to estimate the temperature with respect to time. Using the estimated temperatures, the mean load consumption is predicted for all the consumers located in the modified real-world power grid. Finally using these predicted load consumption values, the margin to collapse scenario is calculated for each node using $\Phi$ from Methods. The color bar indicates the margin to system collapse at each node in the modified real-world power grid. As the (years) increases, the size of each data point in the figure is downscaled.

[20] Marot, A., Donnot, B., Tazi, S. \& Panciatici, P. Expert system for topological remedial action discovery in smart grids (2018).

[21] Marot, A. et al. L2rpn: Learning to run a power network in a sustainable world neurips2020 challenge design (2020).

[22] Marot, A. et al. Learning to run a power network challenge for training topology controllers. Electric Power Systems Research 189, 106635 (2020).

[23] Clarke, L. et al. Scenarios of greenhouse gas emissions and atmospheric concentrations (2007).

[24] National Oceanic and Atmospheric Administration. Historical and forecast api for weather data (2019). URL https://www.weather.gov/documentation/ services-web-api

[25] Riahi, K., Grübler, A. \& Nakicenovic, N. Scenarios of long-term socio-economic and environmental development under climate stabilization. Technological Forecasting and Social Change (2007).

[26] Miller, J. F. \& Howell, D. The ev everywhere grand challenge. World Electric Vehicle Journal (2013).

[27] Weng, Y., Rajagopal, R. \& Zhang, B. A geometric analysis of power system loadability regions. IEEE Trans. on Smart Grid (2019). 